\title{
Isoxanthanol has protective and anti-inflammatory effects on subchondral bone deterioration in experimental osteoarthritic rat model
}

\author{
Xin $\mathrm{li}^{1}$, Jie $\mathrm{Yu}^{1}$, Yan Zhao², Yating $\mathrm{Bai}^{3}$, Lixin Fu${ }^{1}$, Zilong Ma1 ${ }^{1}$ and Shuqin Zhang ${ }^{1 *}$ \\ 'Department of Orthopedics, South of Guang'anmen Hospital, China Academy of Chinese Medical Sciences, Huangcun, Daxing District, Beijing \\ 100053, China; 2Department of Orthopedics, South of Guang'anmen Hospital, China Academy of Chinese Medical Sciences, Huangcun, Daxing \\ District, Beijing 100053, China; '3Department of Emergency, People's Hospital of Beijing Daxing District, Daxing District, Beijing 102600, China
}

In the present study isoxanthanol was investigated for treatment of monosodium iodoacetate (MIA)-induced osteoarthritis (OA) in vivo. The study demonstrated that isoxanthanol inhibited excessive release of interleukin-6, NO and PGE2 in RAW264.7 cells treated with LPS in dose dependent manner. The effects of isoxanthanol were examined in a rat model of osteoarthritis (OA) and observed to ameliorate inflammatory damage and protect against $O A$. Moreover, in vivo data also confirmed inhibition of interleukin-6, NO and PGE2 levels in LPS-induced OA-rats. Deterioration of knee subchondral bone in LPSinduced OA-rats was also prevented effectively by isoxanthanol-treatment. Therefore, isoxanthanol prevents subchondral bone deterioration in $\mathrm{OA}$ rats via targeting inflammatory processes.

Keywords: osteoarthritis, isoxanthanol, subchondral bone, chronic disease, inflammation

Received: 16 March, 2021; revised: 02 July, 2021; accepted: 03 July, 2021; available on-line: 07 February, 2022

凶e-mail: liufengjun6671@yahoo.com

Abbreviations: DMEM, Dulbecco's modified Eagle's medium; IL-6, Interleukin-6; iNOS, Inducible nitric oxide synthetase; MIA, monosodium iodoacetate; NO, Nitric oxide; OA, Osteoarthritis

\section{INTRODUCTION}

Osteoarthritis (OA), a joint disorder that is characterised by degeneration of cartilage, synovitis and altered subchondral bone. This chronic disease is generally caused by the degeneration of weight-bearing joints leading to pain, hindered mobility, and swelling of joints (Hunter \& Bierma-Zeinstra, 2019; Barranco, 2015). Currently joint replacement surgeries are formed for $\mathrm{OA}$ treatment however, these surgeries are very expensive and success rate is also low. The effective chemotherapeutic strategies are yet to be developed. The medications like corticosteroids, nonsteroidal anti-inflammatory drugs (NSAIDs) and hyaluronan preparations are mainly prescribed to the $\mathrm{OA}$ patients for decreasing joint stress or enhancing strength and to inhibit further progression (Barnes and Edwards, 2005). All these therapeutics are accompanied by several adverse effects including kidney damage, bleeding of gastrointestinal tract, and gastrointestinal toxicity (Le Graverand-Gastineau, 2010; Crofford, 2013). Therefore, studies have mostly investigated traditional herbal medicines having no or negligible toxicity for improvement of joint function and controlling joint pain (Cameron \& Chrubasik, 2013; Dhippayom et al., 2015).
$\mathrm{OA}$ at the very onset damages articular cartilage and subchondral bone in the synovial joints followed by changes in pro-inflammatory and anti-inflammatory pathways (Krasnokutsky et al., 2008; Glyn-Jones et al., 2015). Structural changes induced by the OA progression are mainly associated with inflammation (Gong et al., 2012; Musumeci et al., 2012). Hypertrophic chondrocytes have been found to express several biological markers that are involved continuously in the progression of $\mathrm{OA}$ (Vinatier et al., 2016). Progression of OA is associated with the differentiation of chondrocytes, hypertrophic de-differentiation and ultimately leads to apoptosis in a manner similar to that of observed during cartilage ossification. Defect in regulation of chondrocyte growth and deficiency of the differentiation inhibitory factors have been found to promote OA development (Yin et al., 2013). Many inflammatory factors including interleukin (IL) $-1 \beta$ and tumor necrosis factor- $\alpha$ are produced by the chondrocytes which further promote generation of inflammatory factors and consequently change collagen composition and decompose cartilage extracellular matrix (Scanzello et al., 2009). Extracellular matrix decomposition is the major cause of hypertrophy of the chondrocytes (Scanzello et al., 2009). The most useful animal model to study the inflammation and OA for preclinical studies is established by monosodium iodoacetate (MIA) administration (Pitcher et al., 2016). The present study investigated isoxanthanol for treatment of monosodium iodoacetate (MIA)-induced osteoarthritis (OA) in vivo.

\section{MATERIALS AND METHODS}

\section{Cell culture}

The RAW264.7 cell line was provided by the American Type Culture Collection (ATCC, Manassas, VA, USA) and cultured in Dulbecco's modified Eagle's medium (DMEM) at $37^{\circ} \mathrm{C}$ in humidified incubator under $5 \%$ $\mathrm{CO}_{2}$. The medium contained 5.5\% FBS, penicillin (100 $\mathrm{U} / \mathrm{mL})$ and streptomycin $(100 \mathrm{lg} / \mathrm{mL})$.

\section{Analysis of NO production and inflammatory cytokines}

The RAW264.7 cells after pre-treatment with 2 to $16 \mu \mathrm{M}$ doses of isoxanthanol were exposed to LPS $(0.5 \mathrm{lg} / \mathrm{mL})$ for $24 \mathrm{~h}$. The cells were lysed, and the lysate was centrifuged at $12000 \times g$ for $20 \mathrm{~min}$ to collect the supernatant. The supernatant was treated with Griess reagent system (Promega, Madison, WI, USA) and production of $\mathrm{NO}$ was analysed by recording absorbance at 
546 nm. ELISA kits (R\&D Systems, Minneapolis, MN, USA) were used according to manufacturer's instructions to measure the levels of IL-1 $\beta$, IL- 6 and TNF- $\alpha$ in the supernatant.

\section{Western blot}

The RAW264.7 cells after pre-treatment with 2 to $16 \mu \mathrm{M}$ doses of isoxanthanol were exposed to LPS $(0.5$ $\mathrm{lg} / \mathrm{mL}$ ) for $24 \mathrm{~h}$. The cells were harvested by scraping the culture dishes and subsequently lysed for $25 \mathrm{~min}$ on ice in $150 \mu \mathrm{l}$ radioimmunoprecipitation assay buffer containing the protease inhibitors (Pierce; Thermo Fisher Scientific, Inc.). Centrifugation of the lysate at $12000 \times g$ for $10 \mathrm{~min}$ at $4^{\circ} \mathrm{C}$ was followed by collection of the supernatant in which protein concentration was estimated using bicinchoninic acid protein assay kit (Nanjing Keygen Biotech Co., Ltd., Nanjing, China). Protein samples were resolved on $12 \%(\mathrm{w} / \mathrm{v})$ SDS-PAGE and then electroblotted onto polyvinylidene fluoride membranes (Bio-Rad Laboratories, Inc., Hercules, CA, USA). The membranes were blocked for $1 \mathrm{~h}$ at room temperature with $5 \%$ non-fat milk in TBS containing $0.1 \%$ Tween-20 before incubation with primary antibodies for immunodetection at $4{ }^{\circ} \mathrm{C}$ overnight. Incubation of the membranes with goat anti-rabbit horseradish peroxidase (HRP)-conjugated secondary antibody (sc-2004; 1:5000 dilution; Santa Cruz Biotechnology, Inc., Dallas, TX, USA) was carried out for $1 \mathrm{~h}$ at room temperature. The enhanced chemiluminescence substrate to HRP (Invitrogen; Thermo Fisher Scientific, Inc.) was used for determination of the antibody binding. The antibodies used were against: COX-2, iNOS (Cell Signaling, Danvers, MA, USA) and $\beta$-actin (Santa Cruz Biotechnology, Dallas, TX, USA).

\section{Establishment of $O A$ in rats}

Forty Male Sprague-Dawley rats (200-210 g, 7 weeks old) were provided by the Animal Center of Xi'an Jiaotong University (Xi'an, China). The rats were housed under controlled temperature at $23 \pm 2{ }^{\circ} \mathrm{C}$ and $55 \pm 15 \%$ humidity and exposed to $12 \mathrm{~h}$ light/dark cycles. All the rats were given free access to tap water and standard rat chow. Experiments on the rats were conducted in accordance with the guidelines issued by the National Institutes of Health, US. The study was approved by the Institutional Animal Investigation Committee of Xi'an Jiaotong University. Acclimatization for one week was followed by the separation of rats into 4-four groups of 10-each: control group, MIA group and two isoxanthanol (at 5 and $10 \mathrm{mg} / \mathrm{kg}$ ) treatment groups. Solution ( $3 \mathrm{mg} / 50 \mathrm{IL}$ of $0.9 \%$ saline) of MIA was administered directly to the rats into the intra-articular space of the left knee joint. Isoxanthanol at 5 and $10 \mathrm{mg} / \mathrm{kg}$ doses was orally given to the rats in treatment groups in the normal saline one day before administration of MIA. Mixture of ketamine and xylazine was used for anesthetization of the rats and isoflurane overdose was administered for euthanization. Blood samples collected from the rats were centrifuged at $4^{\circ} \mathrm{C}$ for $15 \mathrm{~min}$ at $3000 \mathrm{rpm}$. All the serum samples were stored at $-70^{\circ} \mathrm{C}$ until used for analysis.

\section{Hind paw weight-bearing distribution measurement}

Induction of $\mathrm{OA}$ in rats significantly disrupted the balance in weight-bearing ability of the hind paws. The weight-bearing deficit between the arthritic site to the contralateral limb indicated the index of pain (Combe et al., 2004). Incapacitance tester (Linton Instrumenta- tion, Norfolk, UK) was used for determination of weight bearing of the paw load and inflammatory pain (McDougall et al., 2006).

\section{Histopathology analysis}

Tissues extracted from the knee joints of the rats were fixed in $10 \%$ formalin and then treated with $10 \%$ ethylenediaminetetraacetic acid for decalcification. The tissues were then embedded in paraffin and subsequently sectioned into $7 \mu \mathrm{m}$ slices. The sections were stained using the hematoxylin and eosin (H\&E) or Safranin-O/ Fast Green dye and examined using light microscopy (Olympus BX51, Olympus, Tokyo, Japan) for histological changes.

\section{Real-time PCR analysis}

Total RNA from the knee joint tissues of rats was isolated using TRIzol (Invitrogen, CA, USA). Total RNA $(0.5 \mathrm{mg})$ samples were reverse transcribed into cDNA and one-step RT-PCR kit with SYBR Green (Applied Biosystems, Grand Island, NT, USA) was used for amplification of the PCR. Real-time PCR system (7500, Applied Biosystems) was used for real-time quantitative PCR. The cDNA was subjected to amplification using a TaqManVR Universal PCR master mixture containing DNA polymerase (Applied Biosystems, Foster, CA, USA) as per manufacturer's instructions. Comparative Ct (threshold cycle number at the cross-point between the amplification plot and the threshold) method was used for determination of the concentration of the target genes.

\section{Statistical analysis}

The data presented are the mean \pm standard deviations of the triplicate measurements. The data were analysed statistically using one-way analysis of variance (ANOVA) with Dunnett's multiple comparisons test. All analyses were performed using Prism 7.0 (GraphPad Software, San Diego, CA, USA). Differences were taken as statistically significant at $P<0.05$.

\section{RESULTS}

\section{Isoxanthanol alleviates inflammatory factors}

Stimulation of RAW264.7 cells with LPS was followed by determination of inflammatory response (Fig. 1). LPS-stimulation of RAW264.7 cells led to a significant $(P>0.05)$ elevation of NO and IL- 6 generation compared to control. Production of PGE2 was raised significantly by LPS exposure in RAW264.7 cells. However, on exposure to isoxanthanol LPS-induced NO and IL-6 generation was significantly alleviated in dose-dependent way in 2 to $16 \mu \mathrm{M}$. The LPS-induced PGE2 production in RAW264.7 cells was significantly alleviated on treatment with 2 to $16 \mu \mathrm{M}$ doses of isoxanthanol. In RAW264.7 cells LPS-induced NO and IL-6 generation was reduced to minimum level at $16 \mu \mathrm{M}$ dose.

\section{Isoxanthanol alleviates inflammatory factors}

In RAW264.7 cells LPS induced a prominent increase in iNOS release at $24 \mathrm{~h}$ compared to the control (Fig. 2). Moreover, COX-2 expression also showed a remarkable elevation in RAW264.7 cells on exposure to LPS. However, isoxanthanol treatment caused a significant reduction in iNOS release in RAW264.7 cells. The 
LPS induced excessive COX-2 expression in RAW264.7 cells was also alleviated profoundly on treatment with isoxanthanol.

\section{Isoxanthanol improved weight-bearing distribution of the rat hind paws}

In the MIA administered group weight-bearing distribution of the hind limbs showed a prominent reduction compared to the normal rats (Fig. 3). Reduction in weight-bearing distribution became significant from day one of the MIA administration and continued till 2-weeks. Pre-treatment of MIA administered rats with isoxanthanol at 5 and $10 \mathrm{mg} / \mathrm{kg}$ doses significantly prevented reduction in weight-bearing distribution. Weight-bearing capacity of the hind limbs became completely equal and normal in the MIA administered rats pre-treated with $10 \mathrm{mg} / \mathrm{kg}$ dose of isoxanthanol.

\section{Isoxanthanol alleviates inflammatory response.}

In rats MIA-administration significantly raised the levels of IL-1 $\beta$ and IL- 6 in the serum compared to the normal group (Fig. 4). Moreover, MIA-administration also elevated TNF- $\alpha$ level in the rat serum significantly relative to its content in the normal rat serum. In isoxanthanol pre-treated rats MIA-mediated elevation of IL-1 $\beta$, TNF- $\alpha$ and IL- 6 content in serum was significantly alleviated at 5 and $10 \mathrm{mg} / \mathrm{kg}$ doses. Isoxanthanol pre-treatment lowered the serum content of these inflammatory factors to the level of normal group at 10 $\mathrm{mg} / \mathrm{kg}$ dose.

\section{Isoxanthanol alleviates cytokine mRNA expression in tissues of knee joint}

Administration of MIA to rats caused a drastic elevation of IL-1 $\beta$, TNF- $\alpha$ and IL- 6 mRNA in the knee joint tissues (Fig. 5). The MMP-2 and -9 mRNA levels also showed significant increase in the tissues of knee joint in MIA administered rats. In isoxanthanol pre-treated rats, MIA induced up-regulation of IL-1 $\beta, T N F-\alpha$ and IL-6 mRNA was significantly alleviated in the tissues of knee joints. Isoxanthanol pre-treatment at 5 and 10 $\mathrm{mg} / \mathrm{kg}$ doses also repressed MIA-mediated elevation of MMP-2 and -9 mRNA levels in the rat joint tissues. The COX-2 mRNA level in isoxanthanol pre-treated MIA-administered rats was also lower and comparable to those in the normal group.

\section{Isoxanthanol alleviates knee joint histopathology}

Cartilage damage and the extent of inflammation in the knee joints of MIA-administered rats was analysed using the H\&E staining (Fig. 6). In the joint tissues of MIA-administered rats, pathogenesis of arthritis, infiltration of inflammatory cells in both synovial membrane as well as cartilage were evident. In 5 and $10 \mathrm{mg} / \mathrm{kg}$ isoxanthanol pre-treated rats MIA-mediated pathological changes were significantly inhibited. Pathological alterations by MIA-administration in rat knee joints were completely prevented on pre-treatment with isoxanthanol at $10 \mathrm{mg} / \mathrm{kg}$ dose.

\section{DISCUSSION}

Osteoarthritis is a common disorder of the joints worldwide, hindering movement due to cartilage damage, and there is no clinically approved therapy. It has been established that joint pathology in OA patients is

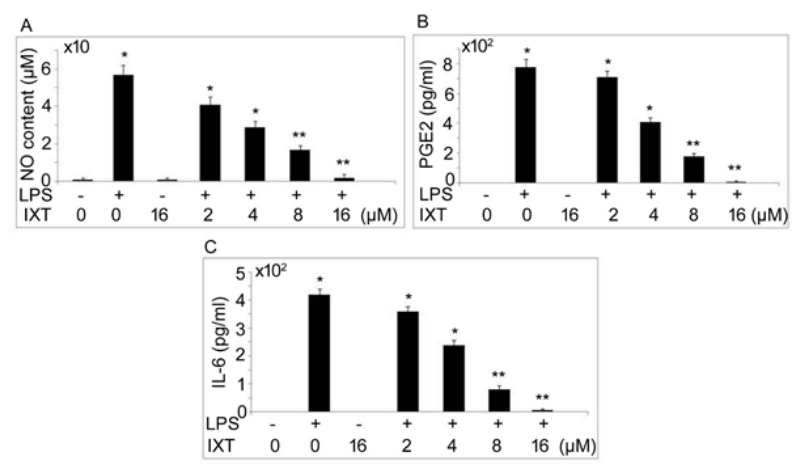

Figure 1. Effect of isoxanthanol on LPS-mediated inflammatory response in RAW264.7 cells.

Pre-treatment with 2 to $16 \mu \mathrm{M}$ doses of isoxanthanol was followed by $24 \mathrm{~h}$ of LPS $(0.5 \mathrm{lg} / \mathrm{mL})$ exposure of RAW264.7 cells. (A) Treatment of the supernatant with Griess reagent was followed by recording absorbance at $546 \mathrm{~nm}$ to measure NO. Production of (B) PGE2 and (C) IL-6 was assessed by ELISA assay. ${ }^{*} P>0.0489$, ${ }^{* *} P>0.0197$ vs. control.

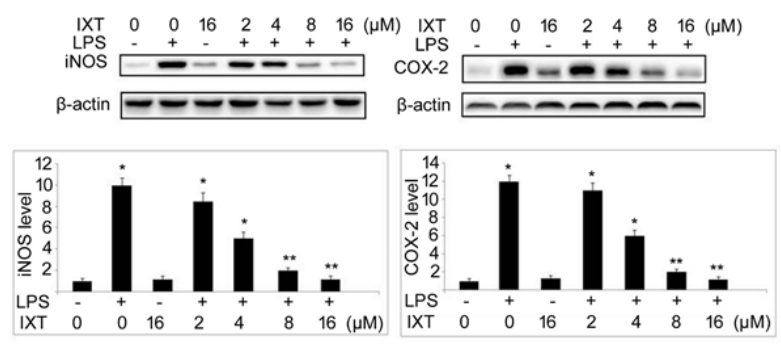

Figure 2. Effect of isoxanthanol on LPS-mediated iNOS release and COX-2 expression in RAW264.7 cells.

Pre-treatment with 2 to $16 \mu \mathrm{M}$ doses of isoxanthanol was followed by $24 \mathrm{~h}$ of LPS $(0.5 \mathrm{lg} / \mathrm{mL})$ exposure of RAW264.7 cells. Western blotting was used to determine iNOS and COX-2 expression. ${ }^{*} P>0.0489,{ }^{* *} P>0.0197$ vs. control.

mainly induced by inflammation, indicating that inhibition of inflammation can act as an effective therapeutic strategy for OA treatment (Philp et al., 2017). Mediators of inflammation like NO and PGE2 as well as cytokines are generated in excess by the inflamed synovium which subsequently degenerate cartilage matrix. Inflammatory response mediated changes result in the appearance of clinical symptoms and degradation of joint cartilages in OA patients (Sellam \& Berenbaum, 2010). Sharp elevation in NO and PGE2 levels during inflammation is directly stimulated by an excessive release of iNOS

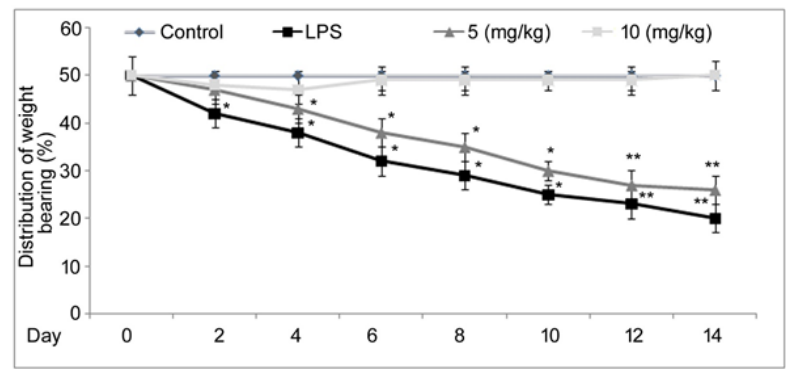

Figure 3. Effects of isoxanthanol on weight-bearing distribution of the rat hind paws.

In the MIA administered rats pre-treated with isoxanthanol at 5 and $10 \mathrm{mg} / \mathrm{kg}$ doses or without pre-treatment the weight-bearing ratio of the rat hind paws was determined after 2-weeks using incapacitance tester. ${ }^{*} P>0.0489,{ }^{*} P>0.0197$ vs. control. 


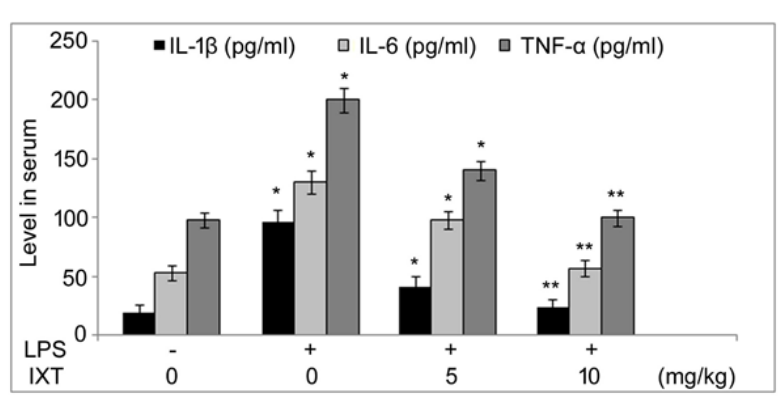

Figure 4. Effects of isoxanthanol on serum cytokines.

In the MIA administered rats pre-treated with isoxanthanol at 5 and $10 \mathrm{mg} / \mathrm{kg}$ doses or without pre-treatment the IL-1 $\beta$, TNF-a and IL- 6 content was analysed in serum by ELISA. ${ }^{*} P>0.0489$, ${ }^{* *} P>0.0197$ vs. control.

and COX-2 (Ahmed et al., 2002; Chabane et al., 2008). Among three isoforms of NOS including eNOS, nNOS, and iNOS, excessive generation of NO during inflammation is induced by iNOS (Lechner et al., 2005). Mediators of inflammation like cytokines and LPS induce increased COX-2 level in many cells and promote PGE2 expression (Parente, 2001; Chun et al., 2004). In the present study stimulation of RAW264.7 cells with LPS led to a significant $(P>0.05)$ elevation of NO and IL-6 generation. Production of PGE2 was also raised significantly by LPS exposure in RAW264.7 cells. However, pre-treatment with isoxanthanol significantly alleviated LPS-induced NO and IL-6 generation in a dose-dependent way at 2 to $16 \mu \mathrm{M}$ concentrations. The LPS-induced PGE2 production in RAW264.7 cells was alleviated significantly on treatment with 2 to $16 \mu \mathrm{M}$ doses of isoxanthanol. In RAW264.7 cells LPS induced a prominent increase in iNOS release at $24 \mathrm{~h}$. Moreover, COX-2 expression also showed a remarkable elevation in RAW264.7 cells on exposure to LPS. Isoxanthanol treatment caused a significant elevation in iNOS release in RAW264.7 cells. The LPS induced excessive COX-2 expression in RAW264.7 cells was also alleviated profoundly on treatment with isoxanthanol. Therefore, isoxanthanol targeted iNOS and COX-2 to suppress excess production of nitric oxide and PGE2. These findings indicate anti-inflammatory potential of isoxanthanol during LPS mediated inflammatory reactions.

During the OA patient's movement is badly reduced and accompanied by severe pain that affects daily physical activities (Bhatia et al., 2013). Key role in cartilage degradation and joint damage is played by the TNF- $\alpha$, IL-1 $\beta$ and IL- 6 which are produced in excess by several cells (Chevalier et al., 2006). Cells of synovial membrane, chondrocytes and osteoblasts besides mononuclear cells that infiltrated into the joints during inflammation play a crucial role in the synthesis of the IL-6 (de Lange-Bro-
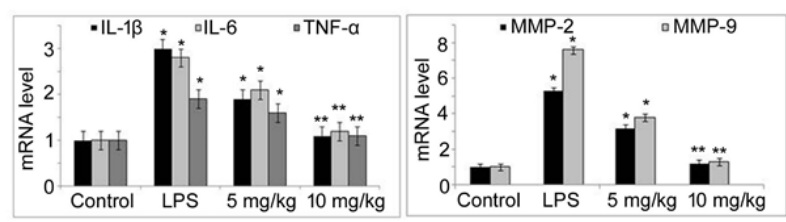

Figure 5. Effect of isoxanthanol on mRNA corresponding to cytokines, mediators of inflammation and MMPS in knee joint tissues.

In the MIA administered rats pre-treated with isoxanthanol at 5 and $10 \mathrm{mg} / \mathrm{kg}$ doses or without pre-treatment the IL-1 $\beta$, COX-2, MMP-2, -9 and IL-6 content was analysed using RT-PCR. ${ }^{*} P>0.0489,{ }^{*} P>0.0197$ vs. control.
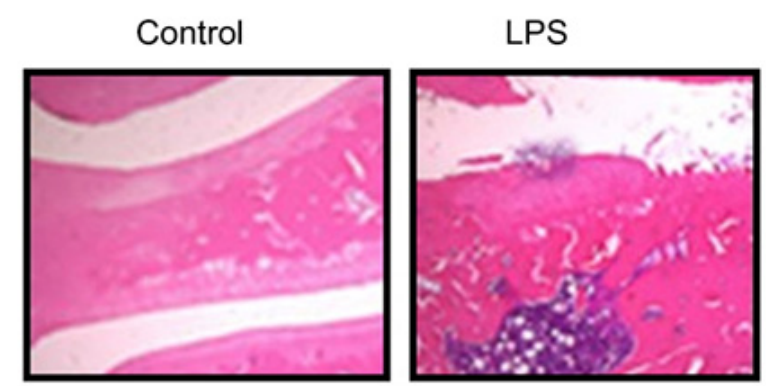

$5(\mathrm{mg} / \mathrm{kg})$

$10(\mathrm{mg} / \mathrm{kg})$

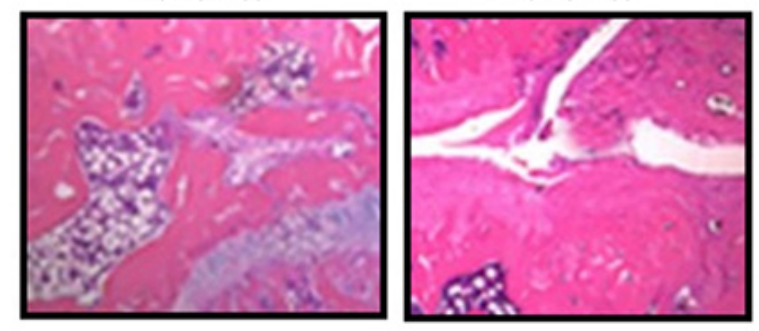

Figure 6. Effect of isoxanthanol on knee pathology.

In the MIA administered rats pre-treated with isoxanthanol at 5 and $10 \mathrm{mg} / \mathrm{kg}$ doses or without pre-treatment the knee pathological alterations were analysed using $H \& E$ staining. ${ }^{*} P>0.0489$, $* * P>0.0197$ vs. control.

kaar et al., 2012). Increased generation of TNF- $\alpha$, IL-1 $\beta$ and IL- 6 in the joint tissues is indicated by higher levels of these cytokines in the synovial fluid and layers of the subchondral bone (Massicotte et al., 2002; Wojdasiewicz et al., 2014). Cascade of events such as cartilage matrix degradation, articular cartilage disintegration, production of ECM proteins and MMPs is stimulated in response to inflammatory cytokines (Meszaros et al., 2015; Zeng et al., 2015). In the present study, MIA administered group weight-bearing distribution of the hind limbs showed a prominent reduction compared to the normal rats. Pretreatment of MIA administered rats with isoxanthanol at 5 and $10 \mathrm{mg} / \mathrm{kg}$ doses significantly prevented reduction in weight-bearing distribution. In rats MIA-administration significantly raised the levels of IL-1 $\beta$ and IL-6 in the serum compared to the normal group. Moreover, MIA-administration also elevated the level of TNF- $\alpha$ in rat serum significantly in relation to its content in normal rat serum. In isoxanthanol pre-treated rats MIA-mediated elevation of IL-1 $\beta$, TNF- $\alpha$ and IL- 6 content in serum was significantly alleviated at 5 and $10 \mathrm{mg} / \mathrm{kg}$ doses. Administration of MIA to rats caused a drastic elevation in IL- $1 \beta$ and IL- 6 mRNA in the tissues of knee joint. The MMP-2 and -9 mRNA levels also showed significant increase in the tissues of knee joint in MIA administered rats. In isoxanthanol pre-treated rats, MIA induced upregulation of IL-1 $\beta$, and IL- 6 mRNA was significantly alleviated in the tissues of knee joints. Isoxanthanol pretreatment at 5 and $10 \mathrm{mg} / \mathrm{kg}$ doses also repressed MIAmediated elevation of MMP-2 and -9 mRNA levels in the rat joint tissues. The COX-2 mRNA level in isoxanthanol pre-treated MIA-administered rats was also lower and comparable to those in the normal group.

Cartilage damage and the extent of inflammation in the knee joints of MIA-administered rats was analysed using the H\&E staining. In the joint tissues of MIAadministered rats, pathogenesis of arthritis, infiltration of inflammatory cells in both synovial membrane as well as cartilage were evident. In 5 and $10 \mathrm{mg} / \mathrm{kg}$ isoxanthanol 
pre-treated rats MIA-mediated pathological changes were significantly inhibited.

\section{CONCLUSION}

In summary, isoxanthanol attenuated LPS-mediated inflammatory response in vitro in RAW264.7 cells. Moreover, LPS mediated joint damage in rats was also prevented effectively on treatment with isoxanthanol. Alleviation of LPS-induced joint damage by isoxanthanol involved inhibition of COX-2, Inos and MMPs. Therefore, isoxanthanol may be developed as a novel therapeutic agent for treatment of OA.

\section{Conflict of interests}

The authors confirm that there are no conflicts of interest.

\section{REFERENCES}

Ahmed S, Rahman A, Hasnain A, Lalonde M, Goldberg VM, Haqqi TM (2002) Green tea polyphenol epigallocatechin-3-gallate inhibits the IL-1 beta-induced activity and expression of cyclooxygenase-2 and nitric oxide synthase- 2 in human chondrocytes. Free Radic Biol Med 33: 1097-1105. https://doi.org/10.1016/s0891-5849(02)010043

Barranco C (2015) Osteoarthritis: Activate autophagy to prevent cartilage degeneration? Nat Rev Rheumatol 11: 127. https://doi. org/10.1038/nrrheum.2015.12

Barnes EV, Edwards NL (2005) Treatment of osteoarthritis. South Med J 98: 205-209. https://doi.org/10.1097/01.smj.0000153116.71823.24

Bhatia D, Bejarano T, Novo M (2013) Current interventions in the management of knee osteoarthritis. I Pharm Bioallied Sci 5: 30-38. https://doi.org/10.4103/0975-7406.106561

Chevalier X, Mugnier B, Bouvenot G (2006) Targeted anti-cytokine therapies for osteoarthritis. Bull Acad Natl Med 190: 1411-1420. PMID: 17450677

Chabane N, Zayed N, Afif H, Mfuna-Endam L, Benderdour M, Boileau C, Martel-Pelletier J, Pelletier JP, Duval N, Fahmi H (2008) Histone deacetylase inhibitors suppress interleukin-1-beta-induced nitric oxide and prostaglandin E2 production in human chondrocytes. Osteoarthritis Cartilage 16: 1267-1274. https://doi.org/10.1016/j. joca.2008.03.009

Combe R, Bramwell S, Field MJ (2004) The monosodium iodoacetate model of osteoarthritis: a model of chronic nociceptive pain in rats? Neurosci Lett 370, 236-240. https://doi.org/10.1016/j.neulet.2004.08.023

Crofford LJ (2013) Use of NSAIDs in treating patients with arthritis. Arthritis Res Ther 15: S2. https://doi.org/10.1186/ar4174

Cameron M, Chrubasik S (2013) Topical herbal therapies for treating osteoarthritis. Cochrane Database Syst Rev 5: CD010538. https://doi. org/10.1002/14651858.CD010538

Chun KS, Cha HH, Shin JW, Na HK, Park KK, Chung WY, Surh YJ (2004) Nitric oxide induces expression of cyclooxygenase-2 in mouse skin through activation of NF-kappaB. Carcinogenesis 25: 445454. https://doi.org/10.1093/carcin/bgh021

de Lange-Brokaar BJ, Ioan-Facsinay A, van Osch GJ, Zuurmond AM, Schoones J, Toes RE, Huizinga TW, Kloppenburg M (2012) Synovial inflammation, immune cells and their cytokines in osteoarthritis: a review. Osteoarthritis Cartilage 20: 1484-1499. https://doi. org/10.1016/j.joca.2012.08.027

Dhippayom T, Kongkaew C, Chaiyakunapruk N, Dilokthornsakul P, Sruamsiri R, Saokaew S, Chuthaputti A (2015) Clinical effects of Thai herbal compress: a systematic review and meta-analysis. Evid Based Complement Alternat Med 2015: 942378. https://doi. org/10.1155/2015/942378
Glyn-Jones S, Palmer AJ, Agricola R, Price AJ, Vincent TL, Weinans H, Carr AJ (2015) Osteoarthritis. Lancet 386: 376-387. https://doi. org/10.1016/S0140-6736(14)60802-3

Gong D, Geng C, Jiang L, Wang L, Yoshimura H, Zhong L (2012) Mechanisms of olive leaf extract-ameliorated rat arthritis caused by kaolin and carrageenan. Phytother Res 26: 397-402. https://doi. org/10.1002/ptr.3567

Hunter DJ, Bierma-Zeinstra S (2019) Osteoarthritis. Lancet 393: 17451759. https://doi.org/10.1016/S0140-6736(19)30417-9

Krasnokutsky S, Attur M, Palmer G, Samuels J, Abramson SB (2008) Current concepts in the pathogenesis of osteoarthritis. Osteoarthritis Cartilage 16: S1-S3. https://doi.org/10.1016/j.joca.2008.06.025

Le Graverand-Gastineau MP (2010) Disease modifying osteoarthritis drugs: facing development challenges and choosing molecular targets. Curr Drug Targets 11: 528-535. https://doi. org/10.2174/138945010791011893

Li G, Yin J, Gao J, et al. (2013) Subchondral bone in osteoarthritis: Insight into risk factors and microstructural changes. Arthritis Res Ther 15: 223. https://doi.org/10.1186/ar4405

Lechner M, Lirk P, Rieder J (2005) Inducible nitric oxide synthase (iNOS) in tumor biology: the two sides of the same coin. Semin Cancer Biol 15: 277-289. https://doi.org/10.1016/j.semcancer.2005.04.004

Massicotte F, Lajeunesse D, Benderdour M, Pelletier JP, Hilal G, Duval N, Martel-Pelletier J (2002) Can altered production of interleukin-1beta, interleukin-6, transforming growth factor-beta and prostaglandin E2 by isolated human subchondral osteoblasts identify two subgroups of osteoarthritic patients. Osteoarthritis Cartilage 10: 491-500. https://doi.org/10.1053/joca.2002.0528

McDougall JJ, Watkins L, Li Z (2006) Vasoactive intestinal peptide (VIP) is a modulator of joint pain in a rat model of osteoarthritis. Pain 123: 98-105. https://doi.org/10.1016/j.pain.2006.02.015

Meszaros EC, Dahoud W, Mesiano S, Malemud CJ (2015) Blockade of recombinant human IL-6 by tocilizumab suppresses matrix metalloproteinase-9 production in the C28/I2 immortalized human chondrocyte cell line. Integr Mol Med 21: 304-310. https://doi. org/10.15761/IMM.1000158

Musumeci G, Carnazza ML, Leonardi R, Loreto C (2012) Expression of b-defensin-4 in 'an in vivo and ex vivo model' of human osteoarthritic knee meniscus. Knee Surg Sports Traumatol Arthrosc 20: 216222. https://doi.org/10.1007/s00167-011-1630-x

Parente L (2001) Pros and cons of selective inhibition of cyclooxygenase-2 versus dual lipoxygenase/cyclooxygenase inhibition: is two better than one? J Rheumatol 28: 2375-2382. PMID: 11708405

Pitcher T, Sousa-Valente J, Malcangio M (2016) The monoiodoacetate model of osteoarthritis pain in the mouse. J Vis Exp 16: e53746. https://doi.org/10.3791/53746

Philp AM, Davis ET, Jones SW, (2017) Developing anti-inflammatory therapeutics for patients with osteoarthritis. Rheumatology (Oxford) 56: 869-881. https://doi.org/10.1093/rheumatology/kew278

Scanzello CR, Umoh E, Pessler F, Diaz-Torne C, Miles T, Dicarlo E, Potter HG, Mandl L, Marx R, Rodeo S, Goldring SR, Crow MK (2009) Local cytokine profiles in knee osteoarthritis: elevated synovial fluid interleukin-15 differentiates early from end-stage disease. Osteoarthritis Cartilage 17: 1040-48. https://doi.org/10.1016/j. joca.2009.02.011

Sellam J, Berenbaum F (2010) The role of synovitis in pathophysiology and clinical symptoms of osteoarthritis. Nat Rev Rheumatol 6: 625-635. https://doi.org/10.1038/nrrheum.2010.159

Vinatier C, Merceron C, Guicheux J (2016) Osteoarthritis: From pathogenic mechanisms and recent clinical developments to novel prospective therapeutic options. Drug Discov Today 21: 1932-1237. https://doi.org/10.1016/j.drudis.2016.08.011

Wojdasiewicz P, Poniatowski LA, Szukiewicz D (2014) The role of inflammatory and anti-inflammatory cytokines in the pathogenesis of osteoarthritis. Mediators Inflamm 2014: 561459. https://doi. org $/ 10.1155 / 2014 / 561459$

Zeng GQ, Chen AB, Li W, Song JH, Gao CY (2015) High MMP-1, MMP-2, and MMP-9 protein levels in osteoarthritis. Genet Mol Res 14: 14811-14822. https://doi.org/10.4238/2015.November.18.46 